\title{
Dynamic anterior cervical plating for multi-level spondylosis: Does it help?
}

\author{
Authors Ashraf A Ragab, F Spain Hodges, Clint P Hill, Robert A McGuire, Michelle Tucci \\ Institution University of Mississippi Medical Center, Jackson, MS, USA
}

\begin{tabular}{|c|c|}
\hline \multicolumn{2}{|l|}{$\begin{array}{l}\text { Methods evaluation and class } \\
\text { of evidence (CoE) }\end{array}$} \\
\hline Methodological principle: & \\
\hline Study design: & \\
\hline RCT & $\bullet$ \\
\hline Cohort & \\
\hline Case control & \\
\hline Case series & \\
\hline Concealed allocation (RCT) & \\
\hline Intent to treat (RCT) & • \\
\hline $\begin{array}{l}\text { Blinded/independent evaluation of } \\
\text { primary outcome }\end{array}$ & $\bullet$ \\
\hline Complete follow-up of $\geq 85 \% *$ & $\cdot$ \\
\hline Adequate sample size & \\
\hline Control for confounding $\dagger$ & $\bullet$ \\
\hline Evidence class: & II \\
\hline $\begin{array}{l}\text { * } \text { Reliable data are data such as mort } \\
\text { reoperation. } \\
\text { † Authors must provide a description } \\
\text { baseline characteristics, and contro } \\
\text { that are unequally distributed betw } \\
\text { treatment groups. }\end{array}$ & those \\
\hline
\end{tabular}

The definiton of the different classes of evidence is available on page 83.

\section{ABSTRACT}

Study design: Randomized controlled trial.

Objective: To compare fusion rates, time to fusion, complication rates and subsidence between 1) a static, 2) a dynamic angulation, and 3) a dynamic translation plate in anterior cervical discectomy and fusion for symptomatic degenerative cervical disease.

Methods: Thirty-six patients with two level, symptomatic cervical degenerative changes requiring surgery were randomized in a blinded fashion to receive a statically locked plate, Cervical Spine Locking Plate (CSLP) (Synthes, Paoli, PN, USA), an Atlantis Vision ${ }^{\circledR}$ Anterior Cervical Plate System (Medtronic, Memphis, TN, USA) which allows angular dynamization, or a Premier ${ }^{\circledR}$ Anterior Cervical Plate System (Medtronic) which allows translational dynamization. Structured data collection and measurement protocols were used. Intervertebral composite allograft cages were used in all groups. Identical external immobilization and antiinflammatory medication protocols were followed. X-rays were obtained at preset time points postoperatively. Assessment of the primary outcomes was blinded. Rate of and time to fusion, graft/instrumentation complications, subsidence, and reoperation for adjacent level disease were measured. Paired t-test and three-way Analysis of Variance test (ANOVA) were used to assess statistical differences between groups.

Results: The three groups were similar demographically. Fusion rates in the CSLP, Atlantis and Premier plate groups were $100 \%, 91 \%$, and $92 \%$ respectively. Mean time to fusion was 6.1 , 8.3 and 6.3 months respectively but differences were not statistically significant. Mean subsidence in the groups was $1.9,1.6$, and $2.6 \mathrm{~mm}$ respectively. Subsidence was found even for the static (CSLP) plate, but no statistically significant differences were found.

Conclusions: We found no clinical advantage of dynamic plates over static plates with regards to fusion rates, time to fusion, subsidence, complications, or adjacent-level surgery. Static plating allows for subsidence at similar levels to dynamic plating. 


\section{STUDY RATIONALE}

Anterior cervical decompression and fusion is considered a gold standard for the treatment of symptomatic spondylosis following failure of appropriate nonsurgical care. Favorable clinical outcomes following anterior cervical discectomy and fusion (ACDF) have been attributed to successful healing/fusion of the interbody graft $[1,2]$. Anterior fixation with plate and screw devices have been recommended for patients requiring multilevel fusions and may play a beneficial role in maintaining or restoring physiologic alignment of an operated neck while assuring a best possible fusion rate, especially when nonautologous structural bone-graft sources are used. Studies have investigated various factors that may enhance fusion rates, including the use of anterior plating $[1,2,3,4]$. Rigid plate and screw implants had been a mainstay of fixation. Questions, however, have been raised whether these implant designs may adversely affect bone healing due to stress shielding and prevention of settling, as has been shown in biomechanical cadaveric studies [5, 6]. These concerns have led to the development of implants with the stated goal of enhancing fusion rates by providing improved load sharing through the anterior interbody grafts and the anterior spinal column. A variety of implant designs have been introduced, some with angulating screws, others with intrinsic mechanisms to allow for compression of structural grafts across the anterior spinal column. These types of implants, however, are invariably more complex and expensive than more conventional rigid locking plates. These "dynamic" implants have been called into question due to their potential to allow for an unwanted collapse and the potential for implant migration. Can the theoretical advantages of dynamization of plates be substantiated in the clinical setting based on radiographic findings? Do "dynamic loading plates" have improved fusion rates and radiographic outcomes compared with conventional rigid locking plates?

\section{OBJECTIVE}

To compare the difference in fusion rates, time to fusion, subsidence levels, complication rates between 1) a static, 2) a dynamic angulation, and 3) a dynamic translation plate and between dynamized plates in general and statically locked plates.

\section{METHODS}

Study design: Randomized controlled trial.

Inclusion criteria: Patients with symptomatic degenerative conditions resulting in radiculopathy or myelopathy who would benefit from a two-level anterior cervical discectomy and fusion (ACDF) with anterior plating.

Exclusion criteria: Exclusion criteria included trauma to the cervical spine, corpectomy, revision surgery, or previous posterior cervical surgery.

\section{Patient population and interventions compared (Fig 1):}

- Of 97 patients who were assessed for eligibility, 61 did not meet inclusion criteria or were excluded as described above. None were lost to follow-up, however, one patient's x-rays could not be adequately measured due to the patient's morbid obesity, leaving 35 patients available for a follow-up rate of $97 \%$ at 12 months.

- All surgeries were performed using the same technique and postoperative regimen by the same surgeon, including use of the same intervertebral allograft cage without use of supplemental graft materials.

- Patients were randomly assigned to one of three plate designs: the cervical spine locking plate (CSLP, Synthes) or the Atlantis (Medtronic); or the Premier (Medtronic). The CSLP plate is a static locked plate that theoretically does not allow settling. The Atlantis and the Premier provided controlled settling.

Device description

\begin{tabular}{lll}
\hline Implant type & $\begin{array}{l}\text { Biomechanical } \\
\text { concept }\end{array}$ & $\begin{array}{l}\text { Device name/ } \\
\text { manufacturer }\end{array}$ \\
\hline Static locking plate & Rigid fixation & $\begin{array}{l}\text { CSLP/Synthes, } \\
\text { Paoli, PN, USA }\end{array}$ \\
\hline Semi rigid & Angular screw & Atlantis/Medtronic, \\
locking plate & transformation in plate & Memphis, TN, USA \\
\hline Translational & Controlled plate & Premier/Medtronic, \\
loading plate & collapse & Memphis, TN, USA \\
\hline
\end{tabular}

- Patients were randomized in blinded fashion where the assistant nurse selected a folded paper which contained the name of the plate from an envelope, without the knowledge of the surgeon.

\section{Outcomes:}

- At follow-up, patients' charts were analyzed for age and gender, smoking status and litigation. If chart data were insufficient, patients were con- 
tacted individually and interviewed via telephone and asked to obtain one last x-ray.

- Only radiographic criteria and measurements were evaluated, as the main purpose of this study was the effect of the plate on radiographic outcome and not clinical outcome. X-rays were evaluated by observers blinded to patient history at 0 and 6 weeks, 3, 6, and 12 months, and at the time of most recent follow-up. X-rays were graded as fused or not fused. Criteria for fusion included the presence of bridging trabeculae across the graft site and the lack of radiolucency between the graft and the adjacent vertebral body.

- Standardized radiographic measurements for settling, plate migration, subsidence and linear translation were used (see web appendix for details). Three independent observers provided the measurements and graded fusion by assessing incorporation of the graft as "healed" or "unclear or not healed". These observers were also asked to asses implant integrity and hardware loosening.

\section{Analysis:}

- Measurements were made from each lateral radiograph to assess 1) fusion rates, 2) time to fusion, 3) subsidence, 4) linear translation, and 5) angular variation [7] (Fig 2).

- After comparison between the three groups was completed, patients with dynamic plates were grouped together (Premier and Atlantis), and compared with the patients with the static plate (CSLP patients).

- Statistical analysis was performed using ANOVA for three-way comparison for the different type of plates. Unpaired Student t-tests were used when the two dynamic plates were grouped and compared against the static plate for the variable values, while chi-square tests were used for categorical values. Results were considered statistically significant when $P<.05$.

Additional detail regarding methods can be found in the web appendix at www.aospine.org/ebsj.

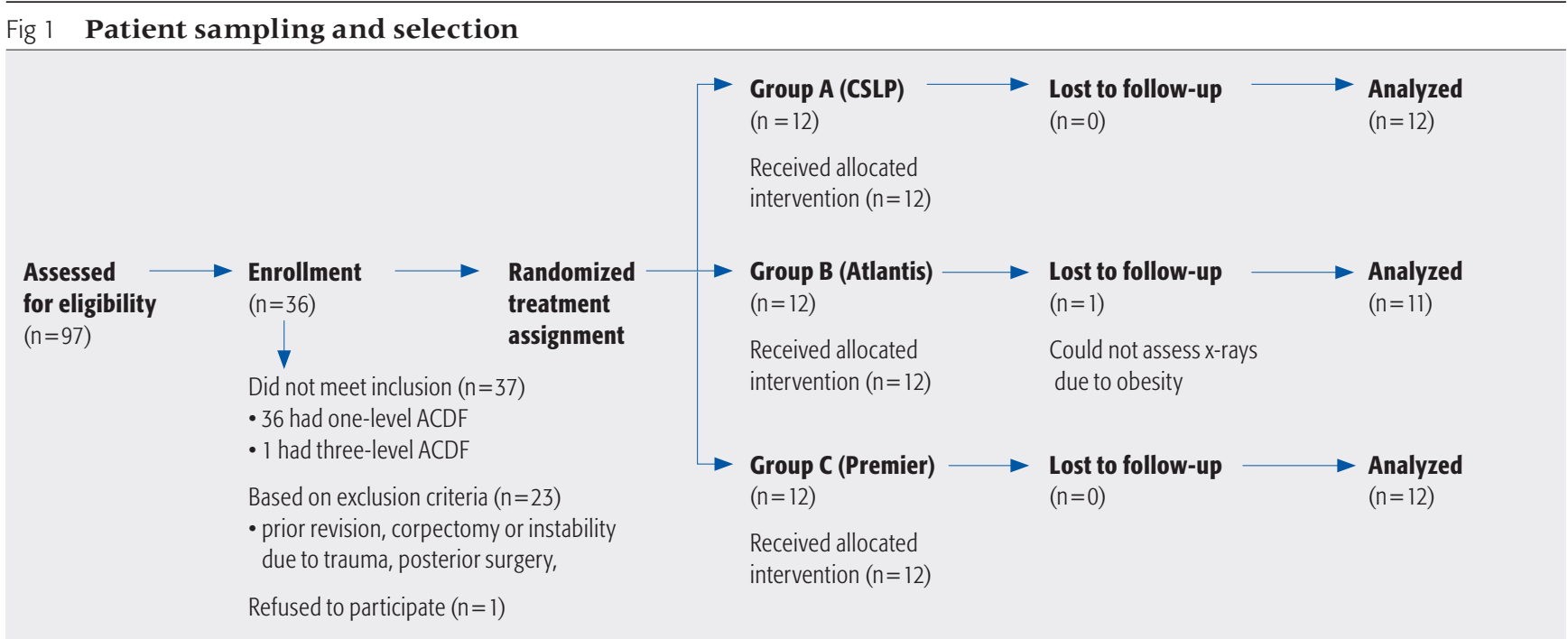

\section{Fig 2 Figure showing points of reference used for measurements.}

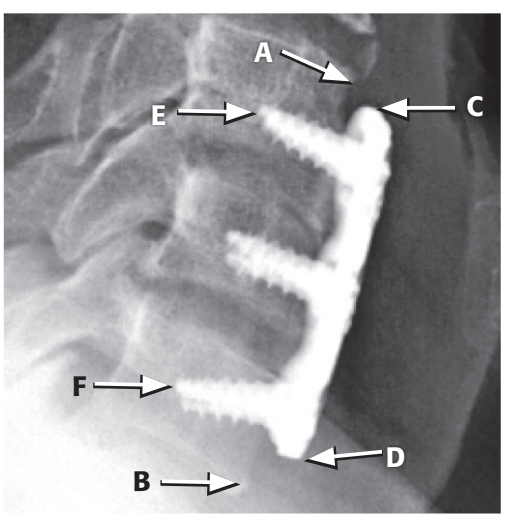




\section{RESULTS}

- No statistical differences with regard to sex, age, follow-up period or smokers were found. (Table 1 and web appendix).

- In the CSLP group, all levels fused. One pseudoarthrosis was found in each of the dynamic plates. These differences were not statistically significant $(P=.61)$.

- No statistical differences between the plates were seen with regard to time to fusion $(P=.59)$, or total subsidence $(P=.63)$ (Table 2 ).

- No statistical differences were found when dynamic plates were grouped together and compared with static plates (Table 3).
- Linear translation or migration differences were found (Table 2). The only statistical difference was found comparing the Premier plate to the Atlantis where $P<.05$. Overlap of the upper end of the plate with the adjacent disc mostly occurred with the Premier plate (Fig 3). To date, none of these patients has required a repeat or adjacent level surgery. Therefore, whether or not a statistical difference was observed, the differences in time to fusion or subsidence were not clinically significant and no patients required any further treatments.

- Angular variation of the upper screws in the Atlantis plate changed from $101.7^{\circ}$ to $98.7^{\circ}$ (mean degrees for the upper and $2.4^{\circ}$ for the middle screws).

\begin{tabular}{llll}
\hline Table 1 & Demographic and baseline characteristics of intervention groups & \\
\hline & $\begin{array}{l}\text { CSLP } \\
\mathbf{N}=\mathbf{1 2}\end{array}$ & $\begin{array}{l}\text { Atlantis } \\
\mathbf{N}=\mathbf{1 1}\end{array}$ & $\begin{array}{l}\text { Premier } \\
\mathbf{N}=\mathbf{1 2}\end{array}$ \\
\hline Age (years) & $58 \pm 15$ & $51 \pm 23$ & $47 \pm 14$ \\
\hline Female (\%) & $8(67 \%)$ & $9(82 \%)$ & $6(50 \%)$ \\
\hline Smokers & $1(1 \%)$ & 0 & 0 \\
\hline Litigation & 0 & 0 & 0 \\
\hline Follow-up (months) & $24(12-42)$ & $29 *(12-49)$ & $22(12-43)$ \\
\hline
\end{tabular}

$\mathrm{N}=$ number enrolled in study

* One patient returned for follow-up 5 years after his surgery.

\section{Table 2 Summary of radiographic findings at last follow-up}

\begin{tabular}{lllll}
\hline & CSLP & Atlantis & Premier \\
& $\mathbf{N = 1 2}$ & $\mathbf{N = 1 1}$ & N=12 & $\boldsymbol{P}$ - value \\
\hline Fusion & $12(100 \%)$ & $10(91 \%)$ & $11(92 \%)$ & .61 \\
\hline Time to fusion(months) & $6.1 \pm 4.5$ & $8.5 \pm 6.5$ & $7.7 \pm 4.8$ & .59 \\
\hline Subsidence (mm) & $1.9 \pm 0.9$ & $2.3 \pm 1.6$ & $2.6 \pm 2.4$ & .63 \\
\hline Linear translation/proximal plate migration $(\mathrm{mm})$ & $2.2 \pm 1.5$ & $1.5 \pm 0.7$ & $3.9 \pm 2.4$ & .018 \\
\hline
\end{tabular}

$N$ reflects the number of patients with data available at last follow-up (after loss to follow-up)

$P$-values are for statistical tests across all three groups. For categorical measures, chi-square analysis was used, and for continuous measures, three way ANOVA was used.

\section{Table 3 Comparison of static plate to dynamic plates grouped together}

\begin{tabular}{llll}
\hline & $\begin{array}{l}\text { Static plate } \\
\mathbf{N = 1 2}\end{array}$ & $\begin{array}{l}\text { Dynamic plates } \\
\text { (grouped) } \mathbf{N = 2 3}\end{array}$ & $\boldsymbol{P}$ - value \\
\hline Fusion & $12 / 12(100 \%)$ & $22 / 24(95 \%)$ & .44 \\
\hline Time to fusion (months) & $6.1 \pm 4.5$ & $8.2 \pm 5.8$ & .32 \\
\hline Subsidence (mm) & $1.9 \pm 0.9$ & $2.1 \pm 1.7$ & .58 \\
\hline Linear translation & $2.2 \pm 1.5$ & $2.8 \pm 2.1$ & .47 \\
\hline
\end{tabular}

$\mathrm{N}$ reflects the number of patients with data available at last follow-up (after loss to follow-up)

$P$-values are for statistical tests across all three groups. For categorical measures, chi-square analysis was used, and for continuous measures, unpaired Student t-tests were used. 


\section{Fig 3a}

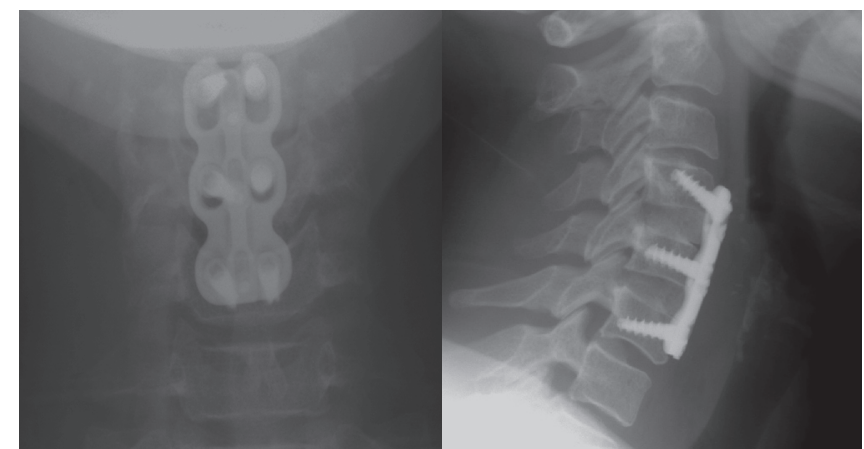

\section{DISCUSSION}

- There does not appear to be a clear clinical advantage of dynamic plates over statically locked plates with regard to fusion rates, subsidence and complication rates.

- In one retrospective comparative study by Du Bois et al. [7], the fusion rate was higher with dynamic plates. In contrast, our study showed a similar fusion rate for all comparison groups. This difference could be attributed to the retrospective, non-randomized study design used by Du Bois, which included one, two and three level surgery, with patients receiving allograft or autograft intervertebral grafts. In our study, these variables were not present.

- Another prospective comparative study [8] did not correlate the fusion rates with the type of plate used. The radiographic correlation was beyond the scope of that study. On the contrary, our study was mainly focused on the radiographic results.

- Strengths: Ours is a randomized controlled trial where potential differences between comparison groups (ie, potential confounding factors) other than those related to anterior fixation choice were kept to a minimum.

- Limitations: The number of patients in each group was small and the study may have been underpowered to detect statistical differences between groups.

- Precise time to fusion would probably require weekly x-rays to be assessed, which would be ethically suspect due to increased radiation exposure and lack of clinical benefit. However, we chose the time points where $\mathrm{x}$-rays are usually obtained in clinical settings, for patient convenience and from a practical stand point. Thus, the exact time results obtained in this study may be limited. Since the same points in time were used for all three plates to assess fusion, our results, however, could be considered a trend or an extrapolation for time to fusion.

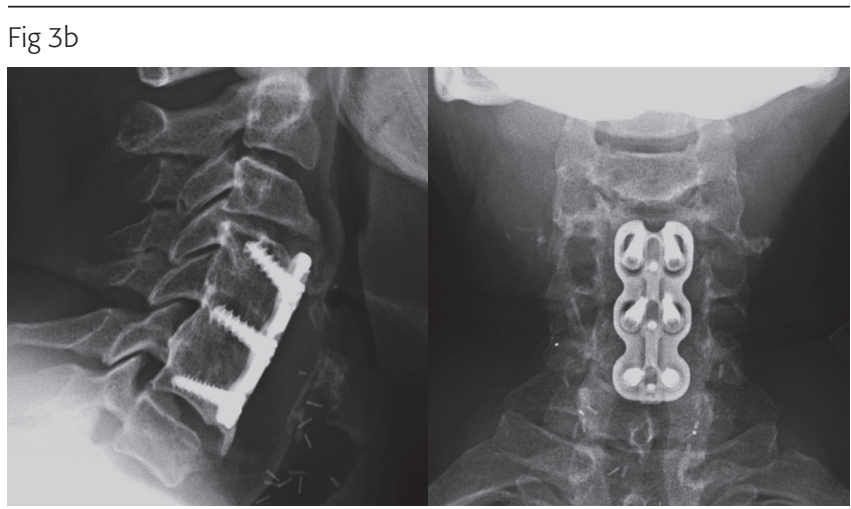

\section{SUMMARY AND CONCLUSIONS}

- This is the first randomized study to compare the radiographic outcomes of three different types of plates (one static and two dynamic) in patients who would benefit from two-level ACDF.

- Use of a dynamic or static plate did not make a difference on any of the radiographic outcomes measured.

- Conclusions drawn from biomechanical studies were not substantiated in this small randomized controlled trial.

- Additional randomized studies with larger sample sizes are needed to further evaluate the benefits of dynamic versus static plating. 


\section{REFERENCES}

1. Bohlman HH, Emery SE, Goodfellow DB, et al (1993) Robinson anterior cervical discectomy and arthrodesis for cervical radiculopathy. J Bone Joint Surg [Am]; 75:1298-1307.

2. Wang J, McDonough P, Endow K, et al (2000) Increased fusion rates with cervical plating for two-level anterior cervical discectomy and fusion. Spine; 25:21-25.

3. Emery SE, Bolesta MJ, Banks MA, et al (1994) Robinson anterior cervical fusion: Comparison of standard and modified techniques. Spine; 19: 660-663.

4. Samartzis D, Shen FH, Lyon C, et al (2004) Does rigid instrumentation increase the fusion rate in one-level anterior cervical discectomy and fusion? Spine J; 4:636-643.

5. Brodke DS, Gollogly S, Alexander Mohr R, et al (2001) Dynamic cervical plates: Biomechanical evaluation of load sharing and stiffness. Spine; 26: 1324-1329.

6. Rapoff AJ, O'Brien TJ, Ghanayem AJ, et al (1999) Anterior cervical graft and plate load sharing. J Spinal Disord; 12: 45-49.

7. DuBois CM, Bolt PM, Todd AG, et al. (2007) Static versus dynamic plating for multilevel anterior cervical discetomy and fusion. Spine $J ; 7(2): 188-193$.

8. Nunley PD, Jawahar A, Kerr EJ, et al (2009) Choice of plate may affect outcomes for single versus multilevel ACDF: results of a prospective randomized single blind trial. Spine J; 9(2): 121-127. 